Random permutations and their discrepancy process 


\title{
The height of watermelons with wall Extended Abstract
}

\author{
Thomas Feierl]
}

Fakultät für Mathematik, Universität Wien, Nordbergstr. 15, A-1090 Wien, Austria

We derive asymptotics for the moments of the height distribution of watermelons with $p$ branches with wall. This generalises a famous result by de Bruijn, Knuth and Rice [2] on the average height of planted plane trees, and a result by Fulmek [6] on the average height of watermelons with two branches.

Keywords: watermelon configuration, vicious walkers, average height, planted plane trees.

\section{Introduction}

The model of vicious walkers was introduced by Fisher [4]. He gave a number of applications in physics, such as modelling wetting and melting processes. In general, the model of vicious walkers is concerned with $p$ random walkers on a $d$-dimensional lattice. In the lock step model, at each time step all of the walkers move one step in any of the allowed directions, such that at no time any two random walkers share the same lattice point.

A configuration that attracted much interest amongst mathematical physicists and combinatorialists is the watermelon configuration, which is a special case of the two dimensional vicious walker model. See Figure 1 for an example of a watermelon, where, for the moment, the broken line labelled 13 should be ignored. This configuration can be studied with or without presence of an impenetrable wall, and with or without deviation. We proceed with a description of $p$-watermelons of length $2 n$ with wall (without deviation), which is the model underlying this paper. Consider the lattice in $\mathbb{R}^{2}$ spanned by the two vectors $(1,1)$ and $(1,-1)$. At time zero the walkers are located at the points $(0,0),(0,2), \ldots,(0,2 p-$ $2)$. The allowed directions for the walkers are given by the vectors $(1,1)$ and $(1,-1)$. Further, the horizontal line $y=0$ is an impenetrable wall, that is, no walker is allowed to cross the $x$-axis. The walkers may now simultaneously move one step in one of the allowed directions, but such that at no time two walkers share the same place. Additionally we demand that after $2 n$ steps all walkers are located at $(2 n, 0),(2 n, 2), \ldots,(2 n+2 p-2)$.

Tracing the paths of the vicious walkers through the lattice we obtain a set of non-intersecting lattice paths with steps in the set $\{(1,1),(1,-1)\}$. In the case of watermelons without deviation, the $i$-th lattice path, also called $i$-th branch, starts at $(0,2 i)$ and ends at $(2 n, 2 i)$. Further, it is seen that the bottom most path is a Dyck path, so that the 1-watermelons with wall correspond to Dyck paths.

\footnotetext{
${ }^{\dagger}$ Research supported by the Austrian Science Foundation FWF, grant S9607-N13.

1365-8050 (c) 2007 Discrete Mathematics and Theoretical Computer Science (DMTCS), Nancy, France
} 


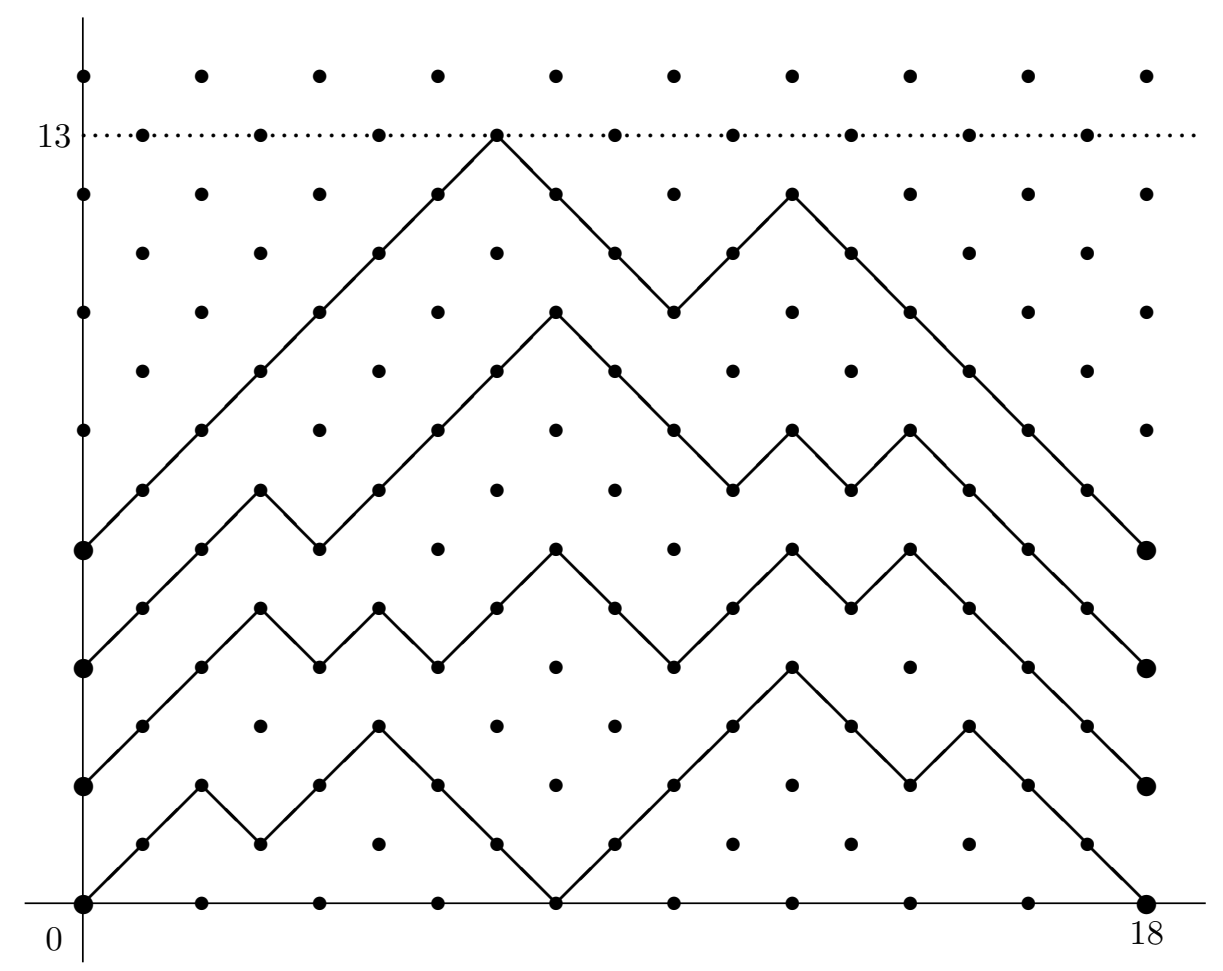

Fig. 1: Example of a 4-watermelon of length 18 with wall and height 13

Since Fisher's introduction [4] of the vicious walkers model numerous papers on this subject have appeared. While early results mostly analyse vicious walkers in a continuum limit, there are nowadays many results for certain configurations directly based on the lattice path description above. For example, Guttmann, Owczarek and Viennot [9] related the star and watermelon configurations to the theory of Young tableaux and integer partitions, and re-derived results for the total number of stars and watermelons without wall. Later, Krattenthaler, Guttmann and Viennot [12] proved new, exact as well as asymptotic, results for the number of certain vicious walkers with wall. Recently, Krattenthaler [11] analysed the number of contacts of the bottom most walker in the case of watermelons with wall, continuing earlier work by Brak, Essam and Owczarek [15].

In 2003, Bonichon and Mosbah [1] presented an algorithm for uniform random generation of watermelons. Amongst other things they used their generator for obtaining experimental results on the height of watermelons. Here, the height of a watermelon is defined as the smallest number $h$ such that the upper most branch does not cross the horizontal line $y=h$. See Figure 1 for an example with four branches and height 13.

As already mentioned, watermelons with one branch are simply Dyck paths. It is well-known that these are in bijection with planted plane trees, and that under this bijection the height of a Dyck path corresponds 
to the height of the corresponding tree. The asymptotic behaviour of the average height of planted plane trees was determined by de Bruijn, Knuth and Rice [2], that is, they solved the average height problem for 1-watermelons with wall. Recently, Fulmek [6] extended their reasoning and determined the asymptotic behaviour of the average height of 2-watermelons with wall.

In this paper we rigorously analyse the height of $p$-watermelons of length $2 n$ with wall, and obtain asymptotics for all moments of the height distribution as $n \rightarrow \infty$. In particular, we show that the $s$-th moment behaves like $\kappa_{s} n^{s / 2}+\kappa_{s-1} n^{(s-1) / 2}+O\left(n^{s / 2-1}+n^{p / 2-p^{2}} \log n\right)$ as $n \rightarrow \infty$ for some explicit numbers $\kappa_{s}$ and $\kappa_{s-1}$, see Theorem 1 at the end of this extended abstract. The nature of our result explains the somewhat inconclusive predictions in [1]. To be more specific, the formulas for the constants $\kappa_{s}$ and $\kappa_{s-1}$ that we find are sums involving coefficients in the Laurent expansion of certain Dirichlet series. It is unlikely that there is any noteworthy simplification in these expressions.

The proof of our result can be summarised as follows. As a first step, we represent the total number of watermelons and the number of watermelons with height restriction in terms of certain determinants (see Lemma 57, the entries being sums of binomial coefficients. From these determinants we then obtain an exact expression for the $s$-th moment of the height distribution. After normalisation we may apply Stirling's formula and obtain an expression that can be asymptotically evaluated using Mellin transform techniques (see Lemma 8). This kind of approach goes back to de Bruijn, Knuth and Rice [2]. Fulmek [6] adopted their approach for the asymptotic analysis of 2-watermelons. The new objects which arise here (and, in general, when extending this approach to the asymptotic analysis of $p$-watermelons) are certain multidimensional Dirichlet series (instead of Riemann's zeta function as in [2]). An additional complication with which one has to cope is the increasing number of cancellations of leading asymptotic terms that one encounters in the calculations while the number $p$ of branches becomes bigger. Thus, while a brute force approach will eventually produce a result for any fixed $p$ (this is, in essence, what Fulmek does for $p=2$ ), the main difficulty that we have to overcome in order to arrive at an asymptotic result for arbitrary $p$ is to trace the roots of these cancellations. We accomplish this with the help of Lemma9 It allows us to exactly pin down which cancellations take place and to extract explicit formulas for the first two terms which survive the cancellations. The multidimensional Dirichlet series which arise in our analysis are the subject of the subsequent section. What we need is information on their poles. This information is obtained with the help of a relation that generalises the reciprocity law for Jacobi's theta functions (see Lemma 2). We note that our definition of these Dirichlet series differs slightly from Fulmek's definition, which makes the analysis somewhat easier. We mention that small modifications immediately yield analogous results for $p$-watermelons with a horizontal wall positioned at some negative integer. Also, the moments for the height distribution of watermelons without wall can be asymptotically determined in a completely analogous fashion.

The paper is organised as follows. The second section contains information on the analytic character of certain multidimensional Dirichlet series that is crucial for the proof of our main result. The third, and last, section contains the main result, see Theorem 1 at the end of that section.

We present all lemmas together with a rough sketch of their proofs, omitting technical details. Complete proofs will be contained in the full paper [3].

We close this section by fixing some notation. Vectors are denoted using bold face letters and are assumed to be $p$-dimensional row vectors. Further, we make use of the 1-norm and the 2-norm of vectors, viz, $|\mathbf{w}|_{1}=w_{0}+\cdots+w_{p-1}$ and $|\mathbf{w}|_{2}^{2}=w_{0}^{2}+\cdots w_{p-1}^{2}$. Finally, we define $\mathbf{v}^{\mathbf{w}}=v_{0}^{w_{0}} \ldots v_{p-1}^{w_{p-1}}$. The relation $\mathbf{v} \geq \mathbf{w}$ is to be understood component-wise. 


\section{Some multidimensional Dirichlet series}

In this section we study the multidimensional Dirichlet series

$$
Z_{\mathbf{a}}(z)=\sum_{\mathbf{m} \neq \mathbf{0}} \frac{m_{0}^{a_{0}} \cdots m_{p-1}^{a_{p-1}}}{\left(m_{0}^{2}+\cdots+m_{p-1}^{2}\right)^{z}}=\sum_{\mathbf{m} \neq \mathbf{0}} \frac{\mathbf{m}^{\mathbf{a}}}{|\mathbf{m}|_{2}^{2 z}},
$$

where $\mathbf{m}=\left(m_{0}, \ldots, m_{p-1}\right)$ ranges over $\mathbb{Z}^{p} \backslash\{0\}$, for $\mathbf{a}=\left(a_{0}, \ldots, a_{p-1}\right) \in \mathbb{Z}^{p}, \mathbf{a} \geq \mathbf{0}$. Our goal is to establish the analytic continuation of $Z_{\mathbf{a}}(z)$ to a meromorphic function and the determination of its poles. Also, we need information on the growth of $Z_{\mathbf{a}}(z)$ as $|z| \rightarrow \infty$ in some vertical strip.

It follows from the definition that $Z_{a_{0}, \ldots, a_{p-1}}(z)=Z_{a_{\sigma(0)}, \ldots, a_{\sigma(p-1)}}(z)$ for every permutation $\sigma \in S_{p}$. If $p=1$ then

$$
Z_{a}(z)=2[a \text { even }] \zeta(2 z-a),
$$

where [Statement] is Iverson's notation, that is

$$
[\text { Statement }]= \begin{cases}1 & \text { if 'Statement' is true, } \\ 0 & \text { otherwise. }\end{cases}
$$

If $a_{p-1}$ is odd, the definition shows that $Z_{a_{0}, \ldots, a_{p-2}, a_{p-1}}(z)=0$. Consequently, we may assume that the parameters $a_{0}, \ldots, a_{p-1}$ are even.

The analytic continuation of $Z_{\mathbf{a}}(z)$ is accomplished very much in the spirit of one of Riemann's methods for the zeta function (see, e.g., [16, Section 2.6]). In fact we have

$$
\frac{(2 \pi i)^{2|\mathbf{a}|_{1}}}{\pi^{z}} Z_{2 \mathbf{a}}(z) \Gamma(z)=\int_{0}^{\infty} t^{z-1}\left(\left(\prod_{j=0}^{p-1} \vartheta_{2 a_{j}}(t)\right)-[\mathbf{a}=\mathbf{0}]\right) d t,
$$

where $\vartheta_{a}(t)=\theta_{a}(0, i t)$ and where

$$
\theta_{a}(x, y)=\frac{\partial^{a}}{\partial x^{a}} \theta(x, y)=(2 \pi i)^{a} \sum_{n=-\infty}^{\infty} n^{a} e^{2 \pi i\left(x n+n^{2} y / 2\right)}
$$

is the $a$-th derivative with respect to $x$ of $\theta(x, y)=\sum_{n} e^{2 \pi i\left(x n+n^{2} y / 2\right)}$, a variant of one of Jacobi's theta functions. Here, Equation (2) is obtained by substitution of Euler's integral for the gamma function and the series definition for $Z_{2 \mathbf{a}}$ on the left hand side of this equation followed by interchanging summation and integration as well as a change of variables in the integral.

We are now going to extract information on the poles of $Z_{2 \mathbf{a}}(z)$ from the integral 22). This task is accomplished with the help of a generalised reciprocity relation (see Corollary 1 , which is a consequence of the following two lemmas. This relation generalises the famous reciprocity law satisfied by Jacobi's theta functions, and is proved following along the lines of the proof of the reciprocity law in [13. Section 2.3].

Lemma 1 Let $\left(f_{a}(x, y)\right)_{a \geq 0}$ be a sequence of functions which are entire with respect to $x$ for every fixed $y$ with $\Im(y)>0$.

If $\left(f_{a}(x, y)\right)_{a \geq 0}$ satisfies the conditions 
(i) $f_{a}(x+1, y)=f_{a}(x, y)$

(ii) $f_{a}(x-y, y)=e^{2 \pi i(x-y / 2)} \sum_{k=0}^{a}\left(\begin{array}{l}a \\ k\end{array}\right) f_{k}(x, y)$

then we have

$$
f_{a}(x, y)=\sum_{k=0}^{a}\left(\begin{array}{l}
a \\
k
\end{array}\right) \frac{c_{0}^{(k)}(y)}{(2 \pi i)^{a-k}} \theta_{a-k}(x, y)
$$

where

$$
c_{0}^{(k)}(y)=\int_{0}^{1} f_{k}(x, y) d x
$$

is the constant term in the Fourier expansion of the function $x \mapsto f_{a}(x, y)$.

Lemma 2 We have

$$
\sum_{k=0}^{\lfloor a / 2\rfloor}\left(\begin{array}{c}
a \\
2 k
\end{array}\right) \frac{(2 k) !}{k !} \pi^{k}\left(\frac{y}{i}\right)^{a-k+1 / 2} \theta_{a-2 k}(x, y)=e^{-i \pi x^{2} / y} \sum_{k=0}^{a}\left(\begin{array}{l}
a \\
k
\end{array}\right)(-x)^{k} i^{k-a}(2 \pi)^{k} \theta_{a-k}\left(\frac{x}{y},-\frac{1}{y}\right) .
$$

Proof Proof (Sketch): The claim can be proved by an application of Lemma 1 to the functions

$$
f_{a}(x, y)=\sum_{n}\left(-\frac{x+n}{y}\right)^{a} e^{-i \pi(x+n)^{2} / y}, \quad a \geq 0 .
$$

Putting $a=0$ in Equation (4), we obtain the reciprocity law for the theta function, viz,

$$
\sqrt{\frac{y}{i}} \theta(x, y)=e^{-i \pi x^{2} / y} \theta\left(\frac{x}{y},-\frac{1}{y}\right) .
$$

Corollary 1 The functions $\vartheta_{a}(y)=\theta_{a}(0, i y), a \geq 0$, satisfy the relation

$$
\vartheta_{a}(y)=i^{a} \sum_{k=0}^{\lfloor a / 2\rfloor}\left(\begin{array}{c}
a \\
2 k
\end{array}\right) \frac{(2 k) !}{k !} \pi^{k}\left(\frac{1}{y}\right)^{a-k+1 / 2} \vartheta_{a-2 k}\left(\frac{1}{y}\right), \quad y>0 .
$$

Proof: The corollary follows from Equation (4) upon setting $x=0$ and replacing $y$ by $i / y$.

We can now state the main result of this section. 
Lemma 3 The function $Z_{2 \mathrm{a}}(z)$ can be analytically continued to a meromorphic function having a single pole of order 1 at $z=\frac{p}{2}+|\mathbf{a}|_{1}$ with residue

$$
\underset{z=\frac{p}{2}+|\mathbf{a}|_{1}}{\operatorname{Res}} Z_{2 \mathbf{a}}(z)=\frac{\pi^{p / 2}}{\Gamma\left(\frac{p}{2}+|\mathbf{a}|_{1}\right)}\left(\prod_{i=0}^{p-1} \frac{\left(2 a_{i}\right) !}{4^{a_{i}} a_{i} !}\right) .
$$

Furthermore, for any non-negative integer $k$ we have

$$
Z_{2 \mathbf{a}}(-k)= \begin{cases}-1 & \text { if } \mathbf{a}=\mathbf{0} \text { and } k=0 \\ 0 & \text { otherwise }\end{cases}
$$

Proof Proof (Sketch): Consider again Equation (2). After splitting the integral above into two parts, one over $[0,1]$ and one over $[1, \infty)$, an application of our generalised reciprocity relation $(5)$ as well as some simple manipulations yield

$$
\begin{aligned}
\frac{(2 \pi i)^{2|\mathbf{a}|_{1}}}{\pi^{z}} Z_{2 \mathbf{a}}(z) \Gamma(z) & =-\frac{[\mathbf{a}=\mathbf{0}] \pi^{z}}{z}+\frac{(-\pi)^{|\mathbf{a}|_{1}} \prod_{j=0}^{p-1} \frac{\left(2 a_{j}\right) !}{a_{j} !}}{z-\frac{p}{2}-|\mathbf{a}|_{1}}+\int_{1}^{\infty} t^{z-1}\left(\left(\prod_{j=0}^{p-1} \vartheta_{2 a_{j}}(t)\right)-[\mathbf{a}=\mathbf{0}]\right) d t \\
& +\int_{1}^{\infty} t^{-z-1}\left(\left(\prod_{j=0}^{p-1} \vartheta_{2 a_{j}}\left(\frac{1}{t}\right)\right)-(-\pi)^{|\mathbf{a}|_{1}}\left(\prod_{i=0}^{p-1} \frac{\left(2 a_{i}\right) !}{a_{i} !}\right) t^{p / 2+|\mathbf{a}|_{1}}\right) d t
\end{aligned}
$$

where the integrals above represent entire functions with respect to $s$. The claims are now easily obtained from this expression.

We close this section with a result on the growth of $Z_{2 \mathbf{a}}(\sigma+i t)$ as $|t| \rightarrow \infty$, which can be proved with the help of [5, Prop. 5].

Lemma 4 For $\sigma \in \mathbb{R}$ fixed we have the estimate

$$
Z_{2 \mathbf{a}}(\sigma+i t)=O\left(e^{\varepsilon|t|}\right), \quad|t| \rightarrow \infty,
$$

for any $\varepsilon>0$.

\section{The moments of the height distribution}

We denote by $M_{2 n, h}^{(p)}$ the number of $p$-watermelons with wall with length $2 n$ and height strictly smaller than $h$. Further, we write $M_{2 n}^{(p)}$ for the total number of $p$-watermelons with length $2 n$. Note that $M_{2 n}^{(p)}=$ $M_{2 n, h}^{(p)}$ for $h \geq n+2 p-1$ and $M_{2 n, h}^{(p)}=0$ for $h<2 p$.

As Bonichon and Mosbah [1], we consider the random variable "height" on the set of $p$-watermelons with wall of length $2 n$ endowed with the uniform probability measure. The goal of this paper is to obtain an asymptotic expression for the $s$-th moment $\mathfrak{m}_{s}^{(p)}(n)$ of this random variable as the length of the watermelons tends to infinity. Clearly, we have

$$
\mathfrak{m}_{s}^{(p)}(n)=\frac{1}{M_{2 n}^{(p)}} \sum_{h \geq 1}\left(h^{s}-(h-1)^{s}\right)\left(M_{2 n}^{(p)}-M_{2 n, h}^{(p)}\right), \quad s \geq 1 .
$$


For determining the asymptotics of $\mathfrak{m}_{s}^{(p)}(n)$ we proceed as follows. First, we obtain determinantal expressions for the quantities $M_{2 n, h}^{(p)}$ and $M_{2 n}^{(p)}$. This is accomplished by an application of a theorem by Lindström-Gessel-Viennot, respectively of a theorem by Gessel and Zeilberger. Second, we obtain asymptotics for

$$
M_{2 n, h}^{(p)} \quad \text { and } \quad \sum_{h \geq 1}\left(h^{s}-(h-1)^{s}\right)\left(M_{2 n}^{(p)}-M_{2 n, h}^{(p)}\right) .
$$

The asymptotics for $\mathfrak{m}_{s}^{(p)}(n)$ are then easily established. The main result is stated in Theorem 1 at the end of this section.

We start with exact expressions for $M_{2 n, h}^{(p)}$ and $M_{2 n}^{(p)}$.

Lemma 5 We have

$$
M_{2 n}^{(p)}=\operatorname{det}_{0 \leq i, j<p}\left(\left(\begin{array}{c}
2 n \\
n+i-j
\end{array}\right)-\left(\begin{array}{c}
2 n \\
n-1-i-j
\end{array}\right)\right)
$$

and

$$
M_{2 n, h}^{(p)}=\operatorname{det}_{0 \leq i, j<p}\left(\sum_{m \in \mathbb{Z}}\left(\begin{array}{c}
2 n \\
n+m(h+1)+i-j
\end{array}\right)-\left(\begin{array}{c}
2 n \\
n+m(h+1)-1-i-j
\end{array}\right)\right), \quad h \geq 0
$$

Proof Proof (Sketch): For $h \geq 2 p$ both equations follow from a theorem by Lindström-Gessel-Viennot (see [7, Corollary 3] or [14, Lemma 1]), respectively from a theorem of Gessel and Zeilberger [8]. To be more specific, Equation (10) follows from the type $C_{p}$ case of the main theorem in [8], while Equation (11) follows from the type $\tilde{C}_{p}$ case.

The reader should observe that the entries of the determinant 10 are the numbers of lattice paths from $(0,2 i)$ to $(2 n, 2 j)$ that do not cross the $x$-axis. On the other hand, the entries of the determinant $[11$, are the numbers of lattice paths from $(0,2 i)$ to $(2 n, 2 j)$ that do not cross the $x$-axis and have height smaller than $h$. These sums are obtained by a repeated reflection principle argument.

For $0 \leq h<2 p$ it is seen that the right hand side of 11$\}$ is equal to zero, as is $M_{2 n, h}^{(p)}$. $\square$ We note that

$$
\begin{aligned}
& \sum_{h \geq 1}\left(h^{s}-(h-1)^{s}\right)\left(M_{2 n}^{(p)}-M_{2 n, h}^{(p)}\right)= \\
& -\sum_{h \geq 1}\left(h^{s}-(h-1)^{s}\right) \sum_{\mathbf{m} \neq \mathbf{0}} \operatorname{det}_{0 \leq i, j<p}\left(\left(\begin{array}{c}
2 n \\
n+m_{i}(h+1)+i-j
\end{array}\right)-\left(\begin{array}{c}
2 n \\
n+m_{i}(h+1)-1-i-j
\end{array}\right)\right)
\end{aligned}
$$

by $(10)$ and $\left[11\right.$, , where the inner sum ranges over $\mathbb{Z}^{p} \backslash\{\mathbf{0}\}$. We now aim at asymptotics for $(9)$. The basic approach to this is as follows. First, we divide the right hand side of $\sqrt{10})$ and $(11)$ by $\left(\begin{array}{c}2 n \\ n\end{array}\right){ }^{p}$. The resulting quotients are then approximated as $n \rightarrow \infty$ with the help of Stirling's formula. It turns out to be convenient to use these approximations in the following form. 
Lemma 6 For $\varepsilon>0$ we have the asymptotic series expansion

$\frac{\left(\begin{array}{c}2 n \\ 2 n+m-z\end{array}\right)}{\left(\begin{array}{c}2 n \\ n\end{array}\right)} \approx e^{-m^{2} / n} \sum_{u \geq 0}\left(-\frac{z}{\sqrt{n}}\right)^{u}\left(\phi_{u}\left(\frac{m}{\sqrt{n}}\right)+\sum_{k=0}^{u-1} \sum_{s>0} n^{-s} \sum_{r \leq 2 s} F_{r, s+r}\left(\begin{array}{c}2 r \\ u-k\end{array}\right) \phi_{k}\left(\frac{m}{\sqrt{n}}\right)\left(\frac{m}{\sqrt{n}}\right)^{2 r+k-u}\right)$

as $n \rightarrow \infty$ uniformly for $m \leq n^{1 / 2+\varepsilon}$ and $z$ bounded. Here, the $F_{r, s+r}$ are some constants the explicit form of which is of no importance in the sequel, and $\phi_{u}(w)$ is defined by

$$
e^{-(m-z)^{2} / n}=e^{-m^{2} / n} \sum_{k \geq 0}(-1)^{k} \phi_{k}\left(\frac{m}{\sqrt{n}}\right)\left(\frac{z}{\sqrt{n}}\right)^{k} .
$$

Hence, we have the explicit expression

$$
\phi_{k}(z)=\sum_{m \geq 0} \frac{(-1)^{m}}{m !}\left(\begin{array}{c}
m \\
k-m
\end{array}\right)(2 z)^{2 m-k}, \quad k \geq 0 .
$$

Asymptotics for $M_{2 n}^{(p)}$ are now easily obtained.

Lemma 7 We have

$$
M_{2 n}^{(p)}=4\left(\begin{array}{c}
p \\
2
\end{array}\right)\left(\prod_{i=0}^{p-1}(2 i+1) !\right)\left(\begin{array}{c}
2 n \\
n
\end{array}\right)^{p} n^{-p^{2}}\left(1+O\left(n^{-1}\right)\right)
$$

as $n \rightarrow \infty$.

Proof: The determinant (10) can be evaluated to closed form, e.g., by means of [10, Theorem 30], and in fact is given by

$$
M_{2 n}^{(p)}=\prod_{j=0}^{p-1} \frac{\left(\begin{array}{c}
2 n+2 j \\
n
\end{array}\right)}{\left(\begin{array}{c}
n+2 j+1 \\
n
\end{array}\right)} .
$$

From this closed form the asymptotics for $M_{2 n, \infty, 1}^{(p)}$ can easily be derived.

For a comprehensive discussion and references of this counting problem we refer to [12, Section 4]

Obtaining the asymptotics for 12 is much harder. It is here where we need the results of the previous section. We begin with the following lemma.

Lemma 8 For $\mathbf{a} \in \mathbb{Z}^{p}, \mathbf{a} \geq \mathbf{0}$, and $k \in \mathbb{N}$ define the function

$$
g_{k, \mathbf{a}}(n)=\sum_{h \geq 1}(h+1)^{k} \sum_{\substack{\mathbf{m} \in(h+1) \mathbb{Z}^{p} \\ \mathbf{m} \neq \mathbf{0}}} e^{-|\mathbf{m}|_{2}^{2} / n}\left(\frac{\mathbf{m}}{\sqrt{n}}\right)^{2 \mathbf{a}} .
$$


For any fixed $M>0$ we have

$$
\begin{gathered}
g_{k, \mathbf{a}}(n)=[p \neq k+1](\zeta(p-k)-1)\left(\prod_{j=0}^{p-1} \frac{\left(2 a_{j}\right) !}{4^{a_{j}} a_{j} !}\right)(n \pi)^{p / 2} \\
+[p=k+1]\left(\left(\prod_{j=0}^{p-1} \frac{\left(2 a_{j}\right) !}{4^{a_{j}} a_{j} !}\right)\left(\gamma-1+\frac{1}{2}\left(\psi\left(p / 2+|\mathbf{a}|_{1}\right)+\log n\right)\right) \pi^{p / 2}+\frac{1}{2} c_{2 \mathbf{a}} \Gamma\left(\frac{p}{2}+|\mathbf{a}|_{1}\right)\right) n^{p / 2} \\
+[p \neq k+1] \frac{1}{2} \Gamma\left(\frac{k+1}{2}+|\mathbf{a}|_{1}\right) Z_{2 \mathbf{a}}\left(\frac{k+1}{2}+|\mathbf{a}|_{1}\right) n^{(k+1) / 2} \\
-\left(B_{k+1} \frac{(-1)^{k}}{k+1}-1\right)[\mathbf{a}=\mathbf{0}]+O\left(n^{-M}\right)
\end{gathered}
$$

as $n \rightarrow \infty$, where $c_{2 \mathbf{a}}$ is the constant term in the Laurent expansion of $Z_{2 \mathbf{a}}(z)$ around its pole and [Statement] is defined by (17).

Proof Proof (Sketch): By the harmonic sum rule, the Mellin transform $g_{k, \mathbf{a}}^{*}(z)$ of $g_{k, \mathbf{a}}\left(x^{-1}\right)$ is seen to be

$$
g_{k, \mathbf{a}}^{*}(z)=\int_{0}^{\infty} g\left(x^{-1}\right) x^{z-1} d x=(\zeta(2 z-k)-1) \Gamma\left(z+|\mathbf{a}|_{1}\right) Z_{2 \mathbf{a}}\left(z+|\mathbf{a}|_{1}\right)
$$

The asymptotic expansion (16) is now obtained upon applying the inverse Mellin transform, pushing the line of integration to the left and taking into account the residues of $g_{k, \mathbf{a}}^{*}(z)$. The displacement of the contour of integration is justified by well known bounds for the gamma and the zeta function together with (7).

$\square$ This last lemma enables us to determine the asymptotics for the non-normalised average (12).

\section{Lemma 9 The sum}

$$
\begin{aligned}
& \sum_{h \geq 1}\left(h^{s}-(h-1)^{s}\right)\left(M_{2 n}^{(p)}-M_{2 n, h}^{(p)}\right)= \\
& -\sum_{h \geq 1}\left(h^{s}-(h-1)^{s}\right) \sum_{\mathbf{m} \neq \mathbf{0}} \operatorname{det}_{0 \leq i, j<p}\left(\left(\begin{array}{c}
2 n \\
n+m_{i}(h+1)+i-j
\end{array}\right)-\left(\begin{array}{c}
2 n \\
n+m_{i}(h+1)-1-i-j
\end{array}\right)\right)
\end{aligned}
$$

satisfies

$$
\begin{aligned}
& \sum_{h \geq 1}\left(h^{s}-(h-1)^{s}\right)\left(M_{2 n}^{(p)}-M_{2 n, h}^{(p)}\right)=2^{-p}\left(\begin{array}{c}
2 n \\
n
\end{array}\right)^{p} n^{-p^{2}} \\
& \times\left(\sum_{\mathbf{a} \geq \mathbf{0}} \operatorname{det}_{0 \leq i, j<p}\left(\frac{(2 i+2 j+2) !}{\left(i+j+1-a_{i}\right) !\left(2 a_{i}\right) !}(-4)^{a_{i}}\right) \tilde{G}_{s, \mathbf{a}}(n)+O\left(n^{s / 2-1}+n^{p / 2-p^{2}} \log n\right)\right)
\end{aligned}
$$


as $n \rightarrow \infty$ for all fixed integers $s$, where

$$
\tilde{G}_{s, \mathbf{a}}(n)=s \tilde{g}_{s-1, \mathbf{a}}(n)-3\left(\begin{array}{l}
s \\
2
\end{array}\right) \tilde{g}_{s-2, \mathbf{a}}(n)
$$

and where

$$
\begin{gathered}
\tilde{g}_{k, \mathbf{a}}(n)=[p \neq k+1] \frac{1}{2} \Gamma\left(\frac{k+1}{2}+|\mathbf{a}|_{1}\right) Z_{2 \mathbf{a}}\left(\frac{k+1}{2}+|\mathbf{a}|_{1}\right) n^{(k+1) / 2}-\left(B_{k+1} \frac{(-1)^{k}}{k+1}-1\right)[\mathbf{a}=\mathbf{0}] \\
+[p=k+1]\left(\left(\prod_{j=0}^{p-1} \frac{\left(2 a_{j}\right) !}{4^{a_{j}} a_{j} !}\right)\left(\gamma-1+\frac{1}{2}\left(\psi\left(p / 2+|\mathbf{a}|_{1}\right)+\log n\right)\right) \pi^{p / 2}+\frac{1}{2} c_{2 \mathbf{a}} \Gamma\left(\frac{p}{2}+|\mathbf{a}|_{1}\right)\right) n^{p / 2} .
\end{gathered}
$$

Here, the quantity $c_{2 \mathrm{a}}$ is defined in Lemma 8 , and [Statement] is defined by 17 .

Proof Proof (Sketch): We consider the more general sum

$$
D_{n}(\mathbf{x}, \mathbf{y}, z)=\sum_{h \geq 1}\left(h^{s}-(h-1)^{s}\right) \sum_{\substack{\mathbf{m} \in(h+1) \mathbb{Z}^{p} \\
\mathbf{m} \neq \mathbf{0}}} \operatorname{det}_{0 \leq i, j<p}\left(\left(\begin{array}{c}
2 n \\
n+m_{i}+x_{i}-y_{j}
\end{array}\right)-\left(\begin{array}{c}
2 n \\
n+m_{i}-z-x_{i}-y_{j}
\end{array}\right)\right) .
$$

We factor $\left(\begin{array}{c}2 n \\ n\end{array}\right)$ out of each row of the determinant above and apply the approximation 13 to all entries of the determinant. This yields a polynomial $P_{N}(\mathbf{x}, \mathbf{y}, z)$. It is seen that this polynomial is divisible by

$$
\left(\prod_{0 \leq i<j<p}\left(x_{i}-x_{j}\right)\left(y_{i}-y_{j}\right)\right)\left(\prod_{0 \leq i \leq j<p}\left(z+x_{i}+x_{j}\right)\left(z+y_{i}+y_{j}\right)\right),
$$

and in fact is of the form

$$
P_{N}(\mathbf{x}, \mathbf{y}, z)=\sum_{\substack{\mathbf{i}, \mathbf{j}, \mathbf{l} \geq \mathbf{0} \\ i_{k}+j_{k}+l_{k} \text { even }}} \frac{\mathbf{x}^{\mathbf{i}} \mathbf{y}^{\mathbf{j}} z^{|\mathbf{l}|}}{n^{(|\mathbf{i}|+|\mathbf{j}|+|\mathbf{I}|) / 2}}\left(\sum_{\mathbf{a} \geq \mathbf{0}} q_{\mathbf{a}, \mathbf{i}, \mathbf{j}, \mathbf{l}}\left(n^{-1}\right) G_{s, \mathbf{a}}(n)\right)
$$

for some polynomials $q_{\mathbf{a}, \mathbf{i}, \mathbf{j}, \mathbf{l}}\left(n^{-1}\right)$ in $n^{-1}$. In principle, the lemma is proved upon inserting the asymptotic expansion 16 for $g_{k, \mathbf{a}}(n)$ above, extracting the coefficient of $\prod_{i=0}^{p-1} x^{2 i+1} y^{2 i+1}$ in $P_{N}(\mathbf{x}, \mathbf{y}, z)$ and verifying that

$$
\sum_{\mathbf{a} \geq \mathbf{0}} q_{\mathbf{a}, \mathbf{v}, \mathbf{w}, \mathbf{l}}\left(n^{-1}\right)\left(\prod_{i=0}^{p-1} \frac{\left(2 a_{i}\right) !}{4^{a_{i}} a_{i} !}\right)=O\left(n^{-(|\mathbf{w}|+|\mathbf{v}|+|\mathbf{1}|) / 2}\right), \quad n \rightarrow \infty .
$$

The verification of this last equation involves the Chu-Vandermonde summation formula. We want to stress that this last fact implies that the first line of 16 does not contribute to the first two terms of the asymptotic expansion 17 .

Finally, we can state the main result of this paper. 
Theorem 1 The s-th moment of the height distribution of p-watermelons with wall satisfies

$$
\begin{aligned}
\mathfrak{m}_{s}^{(p)}=-\frac{2^{-p^{2}}}{\prod_{i=0}^{p-1}(2 i+1) !} \sum_{\mathbf{a} \geq \mathbf{0}} \operatorname{det}_{0 \leq i, j<p}\left(\frac{(2 i+2 j+2) !(-4)^{a_{i}}}{\left(i+j+1-a_{i}\right) !\left(2 a_{i}\right) !}\right) \tilde{G}_{s, \mathbf{a}}(n) & \\
& +O\left(n^{s / 2-1}+n^{p / 2-p^{2}} \log n\right)
\end{aligned}
$$

as $n \rightarrow \infty$, where

$$
\tilde{G}_{s, \mathbf{a}}(n)=s \tilde{g}_{s-1, \mathbf{a}}(n)-3\left(\begin{array}{l}
s \\
2
\end{array}\right) \tilde{g}_{s-2, \mathbf{a}}(n)
$$

and where

$$
\begin{gathered}
\tilde{g}_{k, \mathbf{a}}(n)=[p \neq k+1] \frac{1}{2} \Gamma\left(\frac{k+1}{2}+|\mathbf{a}|_{1}\right) Z_{2 \mathbf{a}}\left(\frac{k+1}{2}+|\mathbf{a}|_{1}\right) n^{(k+1) / 2}-\left(B_{k+1} \frac{(-1)^{k}}{k+1}-1\right)[\mathbf{a}=\mathbf{0}] \\
+[p=k+1]\left(\left(\prod_{j=0}^{p-1} \frac{\left(2 a_{j}\right) !}{4^{a_{j}} a_{j} !}\right)\left(\gamma-1+\frac{1}{2}\left(\psi\left(p / 2+|\mathbf{a}|_{1}\right)+\log n\right)\right) \pi^{p / 2}+\frac{1}{2} c_{2 \mathbf{a}} \Gamma\left(\frac{p}{2}+|\mathbf{a}|_{1}\right)\right) n^{p / 2} .
\end{gathered}
$$

Here, as before, $c_{2 \mathbf{a}}$ is the constant term in the Laurent expansion of $Z_{2 \mathbf{a}}(z)$ around its pole and [Statement] is defined by (1).

Proof: The theorem follows immediately from Lemma 7 and Lemma $9 \quad \square$ For example, our main theorem yields the asymptotics

$$
\begin{aligned}
\mathfrak{m}_{1}^{(1)}=\sqrt{\pi n}-\frac{3}{2}+O\left(n^{-1 / 2} \log n\right), \quad n \rightarrow \infty, \\
\mathfrak{m}_{1}^{(2)}=2.57758 \ldots \sqrt{n}-\frac{3}{2}+O\left(n^{-1 / 2}\right), \quad n \rightarrow \infty, \\
\mathfrak{m}_{1}^{(3)}=3.20787 \ldots \sqrt{n}-\frac{3}{2}+O\left(n^{-1 / 2}\right), \quad n \rightarrow \infty, \\
\mathfrak{m}_{1}^{(4)}=3.74286 \ldots \sqrt{n}-\frac{3}{2}+O\left(n^{-1 / 2}\right), \quad n \rightarrow \infty .
\end{aligned}
$$

Here, numerical approximations for the constants in last three asymptotics were obtained from the exact expressions as given by the last theorem. The first two results are consistent with those in [2] and [6].

\section{References}

[1] N. Bonichon and M. Mosbah. Watermelon uniform random generation with applications. Theoret. Comput. Sci., 307(2):241-256, 2003. Random generation of combinatorial objects and bijective combinatorics.

[2] N. G. de Bruijn, D. E. Knuth, and S. O. Rice. The average height of planted plane trees. In Graph theory and computing, pages 15-22. Academic Press, New York, 1972. 
[3] T. Feierl. The height of watermelons with wall. In preparation.

[4] M. E. Fisher. Walks, walls, wetting, and melting. J. Statist. Phys., 34(5-6):667-729, 1984.

[5] P. Flajolet, X. Gourdon, and P. Dumas. Mellin transforms and asymptotics: harmonic sums. Theoret. Comput. Sci., 144(1-2):3-58, 1995. Special volume on mathematical analysis of algorithms.

[6] M. Fulmek. Asymptotics of the average height of 2-watermelons with a wall. Preprint, available at http://arxiv.org/pdf/math.CO/0607163, 2006.

[7] I. M. Gessel and G. Viennot. Determinants, paths, and plane partitions. Preprint, available at http://people.brandeis.edu/ gessel/homepage/papers/pp.pdf, 1989.

[8] I. M. Gessel and D. Zeilberger. Random walk in a Weyl chamber. Proc. Amer. Math. Soc., 115(1):2731, 1992.

[9] A. J. Guttmann, A. L. Owczarek, and X. G. Viennot. Vicious walkers and Young tableaux. I. Without walls. J. Phys. A, 31(40):8123-8135, 1998.

[10] C. Krattenthaler. Advanced determinant calculus. Sém. Lothar. Combin., 42:Art. B42q, 67 pp. (electronic), 1999. The Andrews Festschrift (Maratea, 1998).

[11] C. Krattenthaler. Watermelon configurations with wall interaction: exact and asymptotic results. $J$. Physics Conf. Series, 42:179-212, 2006.

[12] C. Krattenthaler, A. J. Guttmann, and X. G. Viennot. Vicious walkers, friendly walkers and Young tableaux. II. With a wall. J. Phys. A, 33(48):8835-8866, 2000.

[13] E. Krätzel. Analytische Funktionen in der Zahlentheorie, volume 139 of Teubner-Texte zur Mathematik [Teubner Texts in Mathematics]. B. G. Teubner, 2000.

[14] B. Lindström. On the vector representations of induced matroids. Bull. London Math. Soc., 5:85-90, 1973.

[15] A. L. Owczarek, J. W. Essam, and R. Brak. Scaling analysis for the adsorption transition in a watermelon network of $n$ directed non-intersecting walks. J. Statist. Phys., 102(3-4):997-1017, 2001.

[16] E. C. Titchmarsh. The Theory of the Riemann Zeta-function. The Clarendon Press Oxford University Press, 2 edition, 1986. Edited and with a preface by D. R. Heath-Brown. 
